\title{
Particle spectra at ZEUS
}

\section{Lydia Shcheglova *}

Nuclear Physics Institute of Moscow State University

E-mail: lydia.shcheglova@desy.de

Charged multiplicity distributions and the mean charged multiplicity have been investigated in inclusive neutral current deep inelastic ep scattering with the ZEUS detector at HERA using an integrated luminosity of $38.6 \mathrm{pb}^{-1}$. The measurements were performed in the current region of the Breit frame, as well as in the current fragmentation region of the hadronic center-of-mass frame. The KNO-scaling properties of the data were investigated and the energy dependence of multiplicity distributions was studied using different energy scales. The data are compared to results obtained in $\mathrm{e}^{+} \mathrm{e}^{-}$collisions and to previous DIS measurements as well as to leading-logarithm parton-shower Monte Carlo predictions. The scaled momentum distributions of charged particles in jets have been also measured for dijet photoproduction with the ZEUS detector at HERA using an integrated luminosity of $359 \mathrm{pb}^{-1}$. The distributions are compared to predictions based on perturbative QCD carried out in the framework of the modified leading-logarithmic approximation (MLLA) and assuming local parton-hadron duality (LPHD). The universal MLLA scale, $\Lambda_{\mathrm{eff}}$, and the LPHD parameter, $\kappa^{\mathrm{ch}}$, are extracted.

European Physical Society Europhysics Conference on High Energy Physics

July 16-22, 2009

Krakow, Poland

${ }^{*}$ for the ZEUS Collaboration 


\section{Introduction}

The HERA ep collider provides a rich field for the study of particle production in a wide range of $W$, the photon-proton centre-of-mass (CMS) energy, and the photon virtuality $Q^{2}$. The data presented here were obtained with the ZEUS detector at $\sqrt{s} \sim 300 \mathrm{GeV}^{2}$ and concern the study of the hadronisation and parton fragmentation processes, phenomena which give deep insight into the non-perturbative sector of QCD. Establishing universal features in the properties of the final hadronic system in reactions with different initial particles $\left(\mathrm{e}^{+} \mathrm{e}^{-}\right.$, ep, hadron scattering) helps to elucidate how the partonic cascades evolve into observed hadrons. In particular, recent results on multiplicity distributions in DIS as functions of different energy scales and detailed comparisons with $\mathrm{e}^{+} \mathrm{e}^{-}$are discussed below. The formation of hadron jets was also investigated using the multiplicity and momentum spectra of charged hadrons in the dijet photoproduction events. The measurements verify the validity and consistency of the MLLA approach at energy scale accessed at HERA.

The multipurpose ZEUS detector is described in detail elsewhere [1]

\section{Multiplicity of charged hadrons}

The average multiplicity and multiplicity distributions are being studied intensively in particle collisions. In previous studies of DIS events only the virtuality of the exchanged photon, $Q$, was used as the energy scale [2]. A reasonable agreement with $\mathrm{e}^{+} \mathrm{e}^{-}$data was shown except for the region of $Q$ below 6-8GeV. Recently, the ZEUS collaboration has performed a detailed study of the charged multiplicity in the neutral current deep inelastic scattering (DIS) [3]. These measurements of the charged hadron multiplicity are performed in the Breit and in the hadronic centre-of-mass (HCM) frames. Due to the restricted detector acceptance only hadrons belonging to the current fragmentation regions in both frames were used in the analysis.

\section{ZEUS}

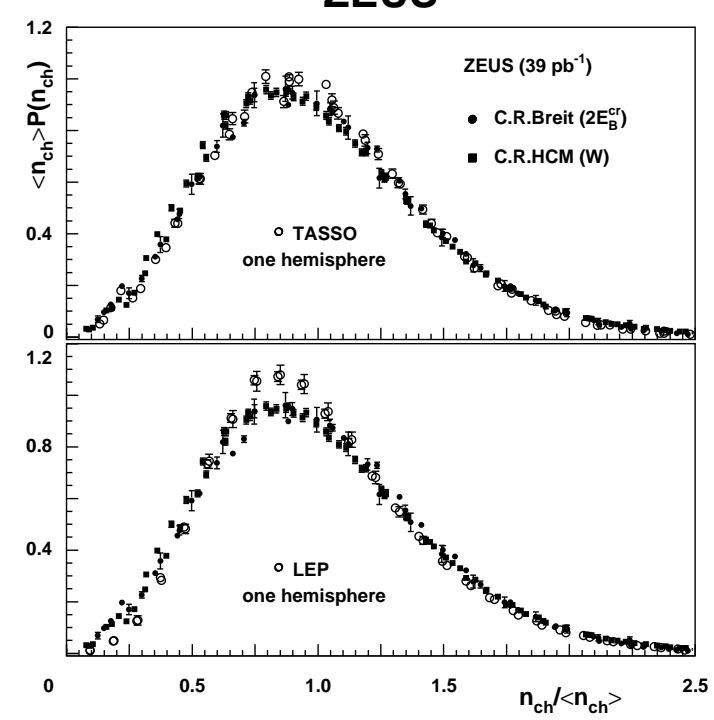

Figure 1: Comparison of multiplicity distributions in $\mathrm{KNO}$ form in bins of $2 \cdot E_{\mathrm{B}}^{\mathrm{cr}}$ and $W$ (solid markers) with $\mathrm{e}^{+} \mathrm{e}^{-}$data for one hemisphere (open circles).

\section{ZEUS}

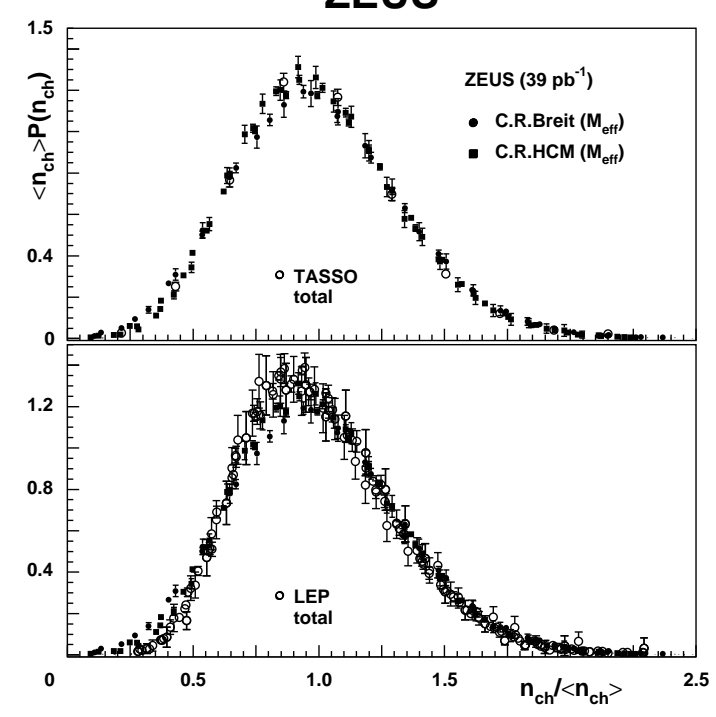

Figure 2: Comparison of multiplicity distributions in KNO form in bins of $M_{\text {eff }}$ (solid markers) with $\mathrm{e}^{+} \mathrm{e}^{-}$data for the whole event (open circles). 
ZEUS investigated charged multiplicity distributions and mean charged multiplicity in terms of different energy scales in order to consistently compare ep DIS data with the data from $\mathrm{e}^{+} \mathrm{e}^{-}, \nu \mathrm{p}$ and $\mu \mathrm{p}$ scattering. The following alternatives to the $Q$ energy scale were considered: the energy of the current region of the Breit frame $E_{\mathrm{B}}^{\mathrm{cr}}$, the invariant mass of the produced particles $W$, used in the current region of HCM, and the invariant mass of the hadronic system $M_{\text {eff }}$, used in both frames.

The scaling properties of multiplicity distributions in a commonly used form, proposed by Koba-Nielsen-Olsen (KNO) [4], were studied in bins of $W, 2 \cdot E_{\mathrm{B}}^{\text {cr }}$ and $M_{\mathrm{eff}}$ and compared with $\mathrm{e}^{+} \mathrm{e}^{-}$data. The multiplicity distributions in the KNO form from ZEUS are shown in Figs. 1. 2. In these plots

ZEUS

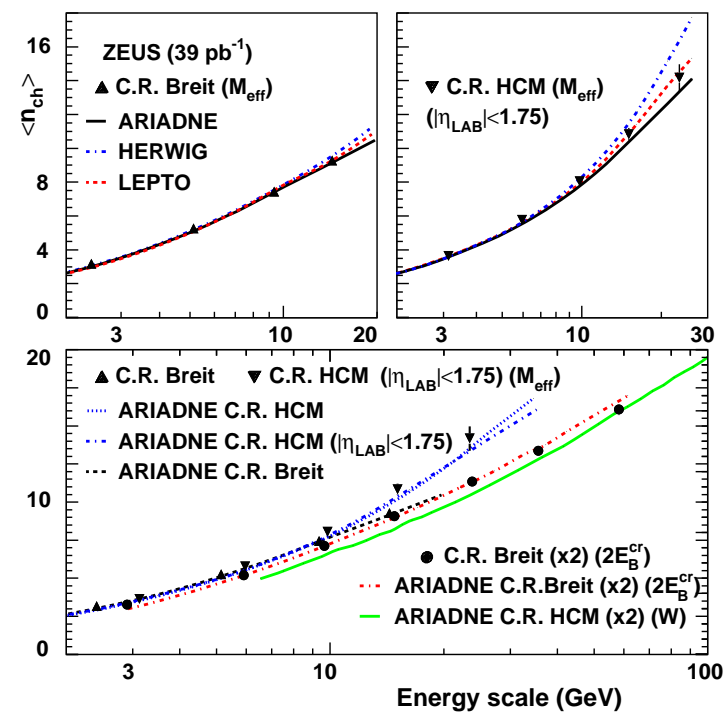

Figure 3: Mean charged multiplicities as a function of $M_{\mathrm{eff}}$ compared to MC predictions (a, b). Comparison of the measurements as functions of $M_{\text {eff }}$ with the measurements as a function of $2 \cdot E_{\mathrm{B}}^{\mathrm{cr}}$ along with the MC predictions (c). the scaled multiplicity distributions, $\Psi(z)=\left\langle n_{\mathrm{ch}}\right\rangle P\left(n_{\mathrm{ch}}\right)$, are plotted as a function of $n_{\mathrm{ch}} /\left\langle n_{\mathrm{ch}}\right\rangle$, where $P\left(n_{\mathrm{ch}}\right)$ and $\left\langle n_{\mathrm{ch}}\right\rangle$ are the multiplicity distribution and average multiplicity respectively. Fig. 1] shows a comparison of the KNO distributions in bins of $2 \cdot E_{\mathrm{B}}^{\mathrm{cr}}\left(12<2 \cdot E_{\mathrm{B}}^{\mathrm{cr}}<100 \mathrm{GeV}\right)$ and in bins of $W(70<W<225 \mathrm{GeV})$ with measurements in one hemisphere of $\mathrm{e}^{+} \mathrm{e}^{-}$, obtained by the TASSO collaboration in the energy range $14<\sqrt{s_{\mathrm{ee}}}<44$ [5] and by the LEP experiments at $\sqrt{s_{\mathrm{ee}}}=91.2 \mathrm{GeV}[6,7]$. There is a remarkable agreement between ep and TASSO data; the LEP data differ somewhat from the present measurement in the peak region and at very low $n_{\mathrm{ch}} /\left\langle n_{\mathrm{ch}}\right\rangle$.

ZEUS

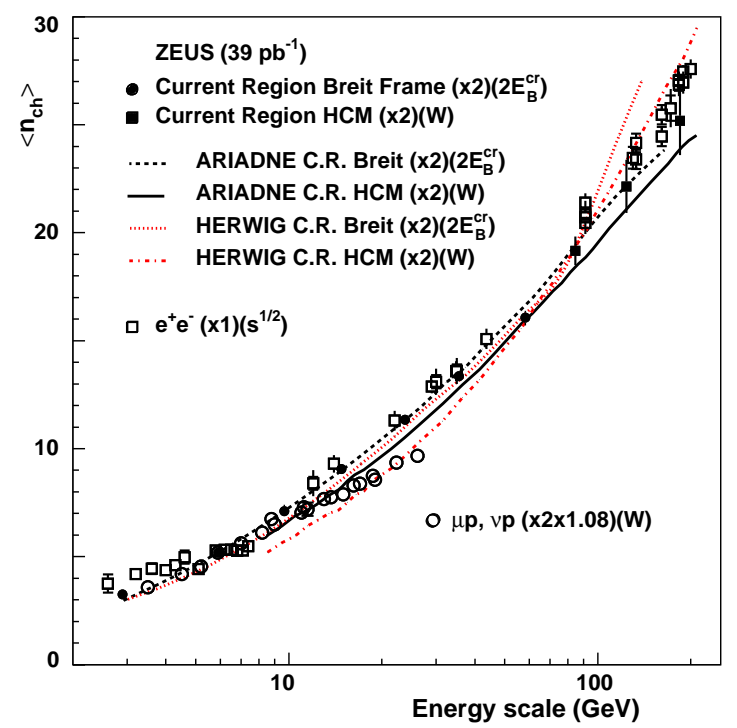

Figure 4: Comparison of mean charged multiplicities as a function of different energy scales with the data from $\mathrm{e}^{+} \mathrm{e}^{-}$and fixed target experiments.
A comparison of the KNO distributions with $\mathrm{e}^{+} \mathrm{e}^{-}$data (both hemispheres) in $M_{\text {eff }}$ bins $\left(8<M_{\text {eff }}<30 \mathrm{GeV}\right)$ in the current region of HCM and in the current region of the Breit frame $\left(8<M_{\mathrm{eff}}<20 \mathrm{GeV}\right)$ is shown in Fig. 2. There is good agreement between the ZEUS and both TASSO [5] and LEP data $\left(91.2<\sqrt{s_{\mathrm{ee}}}<209 \mathrm{GeV}\right)[6-8]$. The mean charged multiplicities were also investigated using the different energy scales discussed above. In Fig. 3 the mean charged multiplicities, measured in the current regions of the Breit and HCM frames, are presented as functions of the invariant mass of the corresponding hadronic system and compared with the MC predictions. All three MC models describe the data reasonably well al- 
though the Herwig prediction is too high in the last bin of $M_{\text {eff }}$ in the current region of the HCM (Figs. 3 (a), (b)). As is seen in Fig. 通 (c), the data in the Breit and HCM frames agree in the region of $M_{\mathrm{eff}}<10 \mathrm{GeV}$, while at higher $M_{\mathrm{eff}},\left\langle n_{\mathrm{ch}}\right\rangle$ rises much faster with $M_{\mathrm{eff}}$ in the current region of HCM than in the current region of the Breit frame. In Fig. B (c) the values of $2 \cdot\left\langle n_{\mathrm{ch}}\right\rangle$ as a function of $2 \cdot E_{\mathrm{B}}^{\mathrm{cr}}$ are also plotted. The data follow the same dependence as $\left\langle n_{\mathrm{ch}}\right\rangle$ vs. $M_{\mathrm{eff}}$ in the Breit frame but differ from those obtained in the current region of the HCM.

Finally, Fig. 4 shows the comparison of the the mean charged multiplicities in the current region of the Breit and HCM frames as a function of $2 \cdot E_{\mathrm{B}}^{\mathrm{cr}}$ and $W$ with the data from $\mathrm{e}^{+} \mathrm{e}^{-}$and fixed-target experiments. The fixed-target data were scaled by a factor 2 (since they were measured in one hemisphere only) and were corrected for the $\mathrm{K}_{\mathrm{S}}^{0}$ and $\Lambda$ decays by a factor 1.08 , estimated using the ARIADNE MC model.The ZEUS measurements show good overall agreement with the data from other experiments and exhibit approximately the same dependence on the respective energy scale; only the fixed target DIS data deviate at energies above $15 \mathrm{GeV}$. The energy scale $2 \cdot E_{\mathrm{B}}^{\mathrm{cr}}$ gives better agreement with $\mathrm{e}^{+} \mathrm{e}^{-}$data at low values of energy than $Q$. The measurements of $\left\langle n_{\mathrm{ch}}\right\rangle$ as a function of $W$ agree, within the uncertainties, with the data from $\mathrm{e}^{+} \mathrm{e}^{-}$collisions.

\section{Scaled momentum distributions}

Recently, momentum spectra of charged hadrons in photoproduction were studied in jet fragmentation processes with the ZEUS detector [9]. The results are compared with perturbative QCD calculations carried out in the framework of the Modified Leading Log Approximation, MLLA, [10] and the hypothesis of Local Parton-Hadron Duality, LPHD [11].The MLLA equations give an analytical description of the parton shower evolution and an effective scale parameter of the QCD calculations, $\Lambda_{\text {eff }}$, that is assumed to be universal, i.e. independent of the process considered. The LPHD hypothesis predicts that the observed hadron distributions should be related to the calculated parton distributions by a normalisation parameter $\kappa^{\mathrm{ch}}$. Tests of the MLLA predictions in conjunction with the LPHD hypothesis permit to expand our understanding of the underlying physics of jet fragmentation phenomenon.

ZEUS

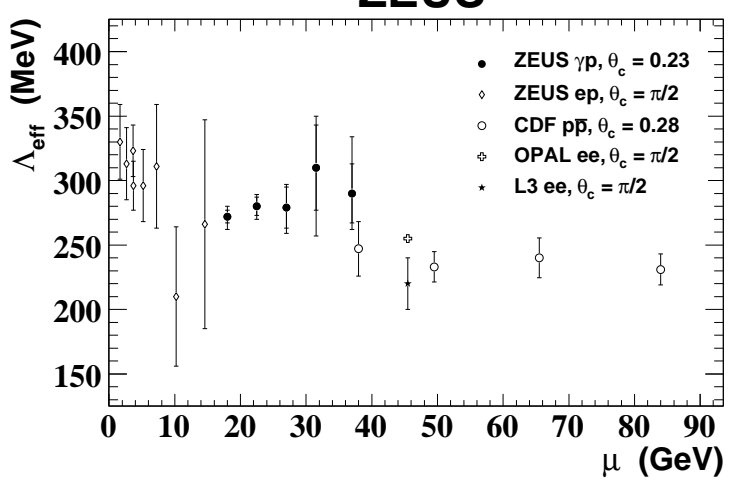

Figure 5: $\Lambda_{\mathrm{eff}}$ as a function of $\mu$, where $\mu$ denotes the characteristic energy scale for each specific process.
Related studies [12] have been performed before in $\mathrm{e}^{+} \mathrm{e}^{-}$collisions at LEP and PETRA, in DIS ep collisions at HERA, (anti)neutrino-nucleon interactions from the NOMAD experiment and $\bar{p} \bar{p}$ collisions at the Tevatron. In the analysis presented here, the momentum spectra of charged hadrons are studied in dijet photoproduction $(\gamma \mathrm{p})$ events from ep collisions. The scaled momentum distributions $\xi=\ln \left(1 / x_{\mathrm{p}}\right)$, where $x_{\mathrm{p}}$ is the fraction of the jet's momentum carried by the charged particle, were measured in restricted cones of various opening angles $\theta_{c}$ around the jet axis. Jets were reconstructed from energy-flow objects [13] (EFOs) by applying the $k_{\mathrm{T}}$ cluster algorithm [14]. The reconstructed invariant dijet mass was used as an energy scale. It probes 
the range 19 to $38 \mathrm{GeV}$, which spans the energy region between those accessed previously by the ZEUS and CDF collaborations.

To check the validity of the MLLA predictions using the measured $\xi$ distributions, two approaches were adopted. The first was based on the position of the peak of the $\xi$ distributions, $\xi_{\text {peak }}$. The second was based on a fit of the full shape of the $\xi$ distributions; the limiting spectra, predicted by MLLA+LPHD theory [10], were used in the fit in this method.

In the $\xi_{\text {peak }}$ analysis the values of $\xi_{\text {peak }}$ were extracted from the $\xi$ distributions using a threeparameter Gaussian fit. At leading order (LO), the peak position is predicted to be at $\xi_{\text {peak }}=\frac{1}{2} Y+\sqrt{c Y}-c$, where $c=0.29$ and $\mathrm{Y}$ is a function of the jet energy $E_{\text {jet }}$ and $\theta_{c}$ (see eq.(3) in [9]) and depends also on the parameter $\Lambda_{\text {eff. }}$. Thus the peak position can be directly fit to the data, treating $\Lambda_{\text {eff }}$ as a free parameter. The best fit value was found to be $\Lambda_{\text {eff }}=275 \pm$ 4(stat.) ${ }_{-8}^{+4}$ (syst.) $\mathrm{MeV}$ for $\theta_{c}=0.23$. In Fig. 5 the values of $\Lambda_{\text {eff }}$ are shown as a function of the energy scale and compared to the results from different experiments. The data are consistent with the prediction that $\Lambda_{\text {eff }}$ is a universal parameter.

The $\xi$ distributions were also fitted using the limiting spectrum predicted by MLLA. The values of $\Lambda_{\text {eff }}$ extracted from these MLLA fits are in reasonable agreement with those extracted from the $\xi_{\text {peak }}$ data, although the values obtained using the MLLA fit have larger uncertainties due to sensitivity of $\Lambda_{\mathrm{eff}}$ to the choice of the fitting range. The values of the LPHD parameters $\kappa^{\text {ch }}$ were extracted also as a function of $E_{\text {jet }}$ and $\theta_{c}$ from the fitted limited momentum spectra and are shown in Fig. 6. The value of $\kappa^{\mathrm{ch}}$, measured with $\theta_{c}=0.23$ and averaged over $E_{\text {jet }}$, was

ZEUS

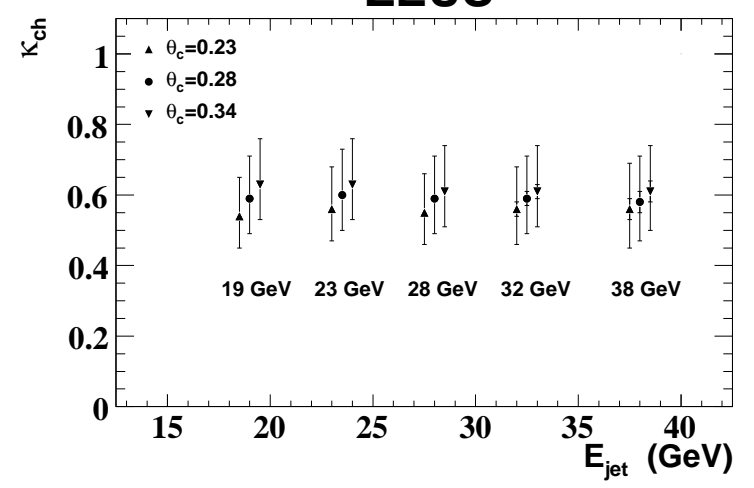

Figure 6: $\kappa^{\text {ch }}$ as a function of $E_{\text {jet }}$ for the three $\theta_{c}$ values.

$\kappa_{\mathrm{ch}}=0.55 \pm 0.01$ (stat. $)_{-0.02}^{+0.03}$ (syst. $)_{-0.09}^{+0.11}$ (theo.) and is in good agreement with that reported by CDF collaboration, $\kappa_{\text {ch }}=0.56 \pm 0.05$ (stat.) \pm 0.09 (syst.). The ZEUS data support the predicted universality of $\kappa_{\mathrm{ch}}$.

\section{Summary and conclusions}

The charged multiplicity distributions and the mean charged multiplicity have been investigated in NC DIS ep scattering in terms of different energy scales. Multiplicity distributions in the scaling $\mathrm{KNO}$ form in the current regions of the Breit and HCM frames exhibit the same behaviour as those in one hemisphere of $\mathrm{e}^{+} \mathrm{e}^{-}$collisions when $2 \cdot E_{\mathrm{B}}^{\mathrm{cr}}$ or $W$ are considered. When energy scale $M_{\text {eff }}$ is used, the charged multiplicities exhibit the same KNO-scaling behaviour as those for the whole $\mathrm{e}^{+} \mathrm{e}^{-}$event. The energy scales $2 \cdot E_{\mathrm{B}}^{\mathrm{cr}}$ and $W$ give better agreement with $\mathrm{e}^{+} \mathrm{e}^{-}$data than $Q$.

The multiplicity distributions of charged particles in dijet photoproduction events have been measured as a function of $\xi=\ln \left(1 / x_{\mathrm{p}}\right)$. Two methods, the $\xi_{\text {peak }}$ analysis and fit of the $\xi$ distributions to the MLLA functions, were used to extract the MLLA scale, $\Lambda_{\text {eff }}$, and LPHD parameter, $\kappa^{\mathrm{ch}}$. The data support the assumption that both parameters are universal. 


\section{Acknowledments}

The author would like to thank my ZEUS collaborators for their efforts to produce the physics results presented at the conference, the ZEUS management for giving her an opportunity to report them here and the organizers for their hospitality.

\section{References}

[1] ZEUS Coll., U Holm (ed.), The Zeus Detector. Status report (unpublished) DESY(1993), available on http://www-zeus.desy.de/bluebook/bluebook.html.

[2] H1 Collaboration, C. Adloff et al., Nucl. Phys. B 504 (1997) 3; ZEUS Collaboration, M. Derrick et al., Z. Phys. C 67 (1995) 93.

[3] ZEUS Collaboration, S. Chekanov et al., JHEP 06 (2008) 061.

[4] Z. Koba, H. B. Nielsen and P. Olesen, Nucl. Phys. B 40 (1972) 317.

[5] TASSO Coll., W. Braunschweig et al., Z. Phys. C 45 (1989) 193.

[6] DELPHI Coll., P. Abreu et al., Z. Phys. C 50 (1991) 185.

[7] OPAL Coll., P. D. Acton et al., Z. Phys. C 35 (1991) 539.

[8] see Chekanov et al., JHEP 06 (2008) 061 and ref. [37] ibidem.

[9] ZEUS Collaboration, S. Chekanov et al., JHEP 08 (2009) 077.

[10] see S. Chekanov et al., JHEP 08 (2009) 077 and ref. [1] ibidem.

[11] Y. I. Azimov et al., Z. Phys. C 27 (1985) 65.

[12] see S. Chekanov et al., JHEP 08 (2009) 077 and refs. [3-9] ibidem.

[13] ZEUS Collaboration, J. Brietweg et al., Eur. Phys. J. C 6 (1999) 43.

[14] S. Catani et al., Nucl. Phys. B 406 (1993) 187. 